\title{
legless insertional mutation: morphological, molecular, and genetic characterization
}

\author{
Gurparkash Singh, Dorothy M. Supp, Claire Schreiner, John McNeish ${ }^{1}$ H.-J. Merker, ${ }^{2}$ \\ Neal G. Copeland, ${ }^{3}$ Nancy A. Jenkins, ${ }^{3}$ S. Steven Potter, ${ }^{4}$ and William Scott \\ Children's Hospital Medical Center, Department of Pediatrics, University of Cincinnati College of Medicine, \\ Cincinnati, Ohio 45229 USA; $^{1}$ Department of Pathology, University of North Carolina, Chapel Hill, North Carolina 27599 ; \\ ${ }^{2}$ Institut für Toxikologie und Embryonalpharmakologie, Freie Universität Berlin, D-1000 Berlin 33, Germany; ${ }^{3}$ Mammalian \\ Genetics Laboratory, ABL-Basic Research Program National Cancer Institute Frederick Cancer Research and Development \\ Center, Frederick, Maryland 21702 USA
}

Limb morphogenesis is an excellent model system to study pattern formation during vertebrate development. The legless ( $(g l)$ insertional mutation can serve as a tool to analyze specific events in limb development at the embryologic, genetic, and molecular levels. Hemizygous mice of this transgenic line are phenotypically normal, but homozygous mutants are inviable and exhibit limb, brain, and craniofacial malformations, as well as situs inversus. By morphological analysis of mutant hindlimb buds we show absence of a normal apical ectodermal ridge, a structure required for limb bud outgrowth, and an unusually high degree of mesenchymal and ectodermal cell death. Mutant embryos are extremely sensitive to retinoic acid, a known teratogen with a proposed role in limb development. The hindlimb malformations in legless mutants are less severe when bred into the BALB/c background, suggesting the involvement of other strain-specific genes. Molecular analysis of the disrupted region indicates two tightly linked insertion sites. Sequences flanking the transgene insertions have been cloned and mapped to chromosome 12, near the iv (situs inversus viscerum) locus. Consistent with this, complementation tests confirm allelism of $l g l$ and $i v$ and suggest that the transgene insertion may have disrupted more than one gene. Phylogenetically conserved sequences flanking the transgene insertions were identified and used to isolate candidate $l g l$ and iv cDNAs.

[Key Words: Insertional mutation; limb development; visceral asymmetry; iv locus and transgenic mice]

Received July 17, 1991; revised version accepted September 13, 1991.

The vertebrate limb has served as a powerful model system for the investigation of pattern formation during development. The process of limb formation is well conserved during evolution, with grafted pieces of limb buds from mammals or reptiles capable of driving appropriate chicken limb development (Fallon and Crosby 1977). Furthermore, limb morphogenesis has been hypothesized to use a number of interesting mechanisms, including gradients emanating from the zone of polarizing activity (ZPA) (Tickle et al. 1975), cell division memory in the mesenchymal progress zone (Summerbell and Lewis 1975), reaction-diffusion processes (Turing 1952), and essential, although poorly understood, inductive interactions between the mesenchyme and apical ectodermal ridge (AER) (Harrison 1918; Zwilling 1955; Saunders et al. 1957; Hurle et al. 1989). To unravel limb development at the molecular level it is necessary to characterize the key genetic components that govern the process.

Transgene insertional mutagenesis can randomly scan

${ }^{4}$ Corresponding author. the mammalian genome, searching for genes of developmental significance. Conceptually, the approach is identical to the transposable element tagging that has been used so successfully in identifying interesting genes in bacteria (Heffron et al. 1975), Drosophila (Rubin et al. 1982), Caenorhabditis elegans (Moerman et al. 1986), and plants (O'Reilly et al. 1985). In each case, the foreign DNA serves as both mutagen and molecular tag, allowing molecular retrieval of the altered chromosomal region.

Previously, we have described the developmentally interesting phenotype observed in newborn mice homozygous for the legless (Igl) transgene insertional mutation, which arose in the pHT1-1 line of transgenic mice (McNeish et al. 1988, 1990). As the name suggests, essentially all of these mice have deficient hindlimbs, with structures distal to the femur absent. Furthermore, the forelimbs are also malformed, but with a markedly variable expressivity. Some forelimbs are only mildly affected, with missing anterior digits; other forelimbs are severely altered, with the paws and anterior forearm 


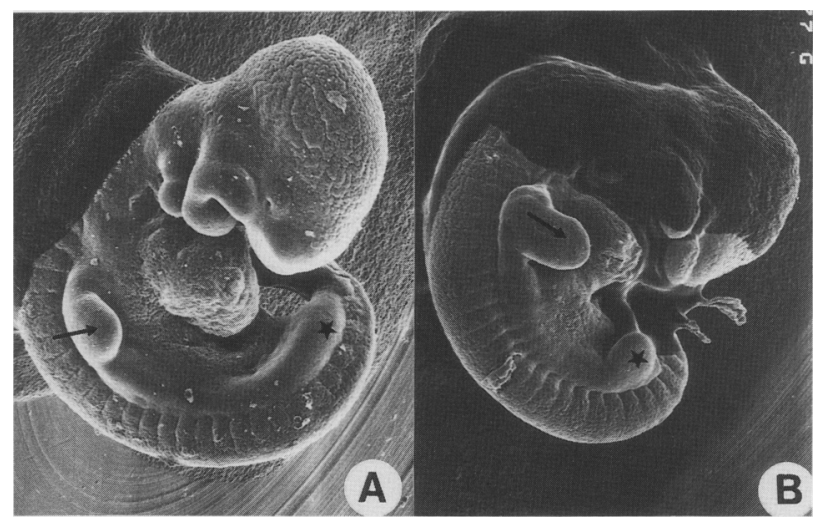

Figure 1. Normal development of forelimb (arrow) and hindlimb $\left({ }^{*}\right)$ buds in $1 g 1 / 1 \mathrm{gl}$ embryos through day 10 of gestation. $\langle A|$ An early day 10 embryo with 28 somites. (B) A late day 10 embryo with 40 somites. Magnification, $16.8 \times$.

bone (the radius) missing, but with the posterior forearm bone (the ulna) present and normal. That is, $1 g l / 1 g l$ mice are lacking anterior, distal forearm structures, but with considerable individual variation. Moreover, the mutant mice have aberrant brains, with the most anterior structures, the olfactory lobes, usually absent. The cerebral hemispheres are often malformed, with hydrocephaly, encephaloceles, and darkened necrotic regions, but the more posterior structures of the mutant brain are normal. Craniofacial clefts are observed in $\sim 50 \%$ of the mutants. And finally, in half of the mutant mice we observed situs inversus, a mirror image arrangement of internal organs. This suggests that a gene product that normally governs visceral positioning is now absent or defective, allowing randomization of internal left-right asymmetry.

In this report we describe the results of our continued embryologic, genetic, and molecular studies of this insertional mutation. During early hindlimb morphogenesis we observed the absence of normal AER, as well as unusual mesenchymal cell death. Genetic background effects were investigated by backcrossing to the BALB/c inbred mouse strain, resulting in distinct modifications of the phenotype. Furthermore, mutant embryos were shown to be remarkably sensitive to retinoic acid. At the molecular level, mouse DNA flanking the transgene insertion has been cloned and used for chromosomal mapping. The results suggested allelism with the classic iv (situs inversus viscerum) locus, and this was confirmed by genetic complementation tests. In addition, we have demonstrated that the transgene disruption was complex in nature, likely involving two tightly linked transgene insertions. Two mouse flanking DNA segments with highly evolutionarily conserved sequences were localized and used to retrieve candidate $1 g l$ gene cDNAs.

\section{Results}

Developing hindlimbs

Hindlimb buds of $l g l / l g l$ and wild-type embryos were ex- amined by scanning electron microscopy (SEM) to search for early morphologic lesions that might underlie the subsequent phenotype. Hindlimbs were ideal for this analysis because the uniformity of the mutant phenotype allowed the association of an observed embryologic defect with a known outcome. Figure 1 shows that early hindlimb bud development in $1 g l / l g l$ embryos progresses within normal limits through day 10 of gestation. These are both mutant embryos, but $l g l / l g l$ hindlimb bud development at this stage cannot be distinguished from controls. By day 11 , the mutant hindlimb buds failed to develop to the paddle stage achieved by wild-type embryos (Fig. 2). This lack of outgrowth is most likely the result of absence of the AER. Figure 2, A and B, illustrates the AER in hindlimb buds from day 11 wild-type and hemizygous mouse embryos. The absence of this structure in comparably aged mutant hindlimb buds is evident in Figure 2, C and D.

\section{Histologic analysis}

Serial histologic sections of hindlimb buds from embryos of 28-40 somites verified the SEM findings, with no AER detected in mutants. In the early day 10 embryos $(28-30$

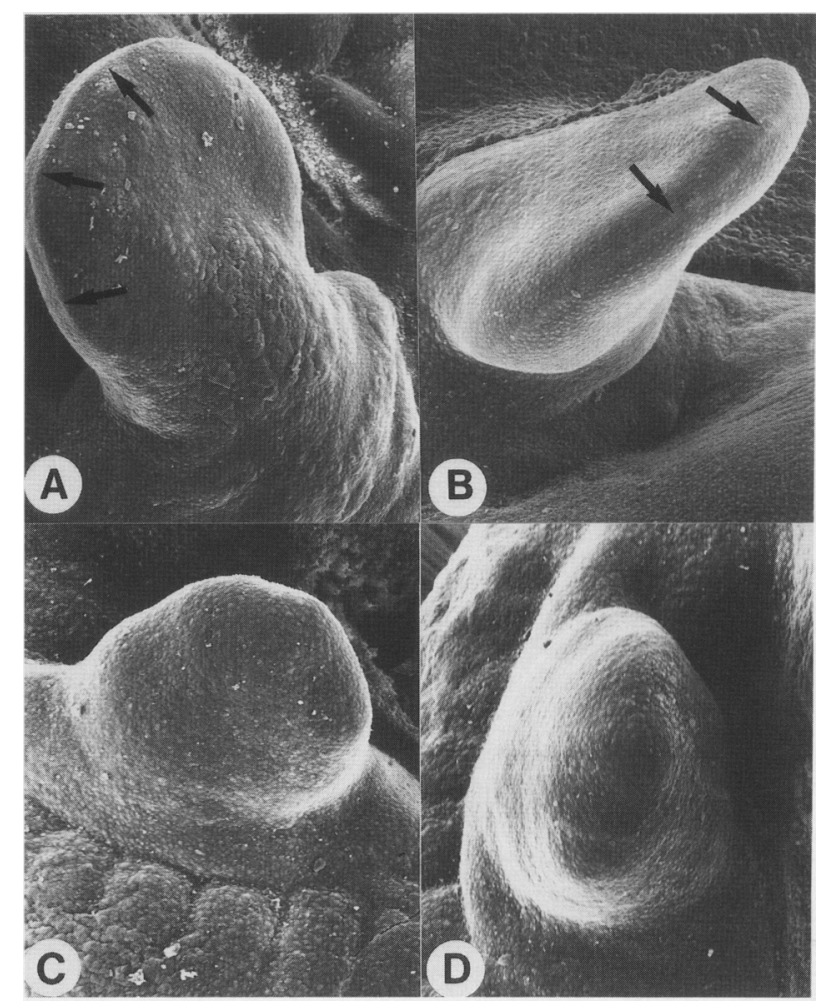

Figure 2. SEM of day 11 hindlimb bud. (A) Day 11 wild-type embryo. Arrows indicate AER. (B) Hindlimb bud from day 11 $1 g l /+$ embryo. Arrows indicate AER. $(C, D)$ Different views of the hindlimb bud from day $11 \mathrm{lgl} / \mathrm{lgl}$ embryo. The bud is growth retarded, has not attained the characteristic paddle shape, and is lacking an AER. Magnification, $47 \times$. 
somites|, however, no differences could be discerned from wild-type littermates. The first noticeable difference in mutant embryos appears at 34-35 somites as an episode of necrosis in the anterior (preaxial) mesoderm in the dorsal half of the hindlimb bud (Fig. 3). As the mutant embryo reaches the 40 -somite stage, mesodermal cell death becomes more intense and extends throughout the limb mesoderm, especially in the dorsal half of the bud (Fig. 4). Surprisingly, mitotic figures can be found in the midst of this necrotic episode. At this stage, cell death in the ectoderm becomes evident and involves the entire anteroposterior extent of apical ectoderm (Fig. 4). This episode of cell death begins somewhat more ventrally than the mesodermal necrosis. In the day $11 \mathrm{mu}$ tant hindlimb (45-50 somites), debris from dying cells in the mesoderm is minimal and the ectoderm is generally free of cell death but shows no thickening indicative of AER. One exception was noted where a small, localized

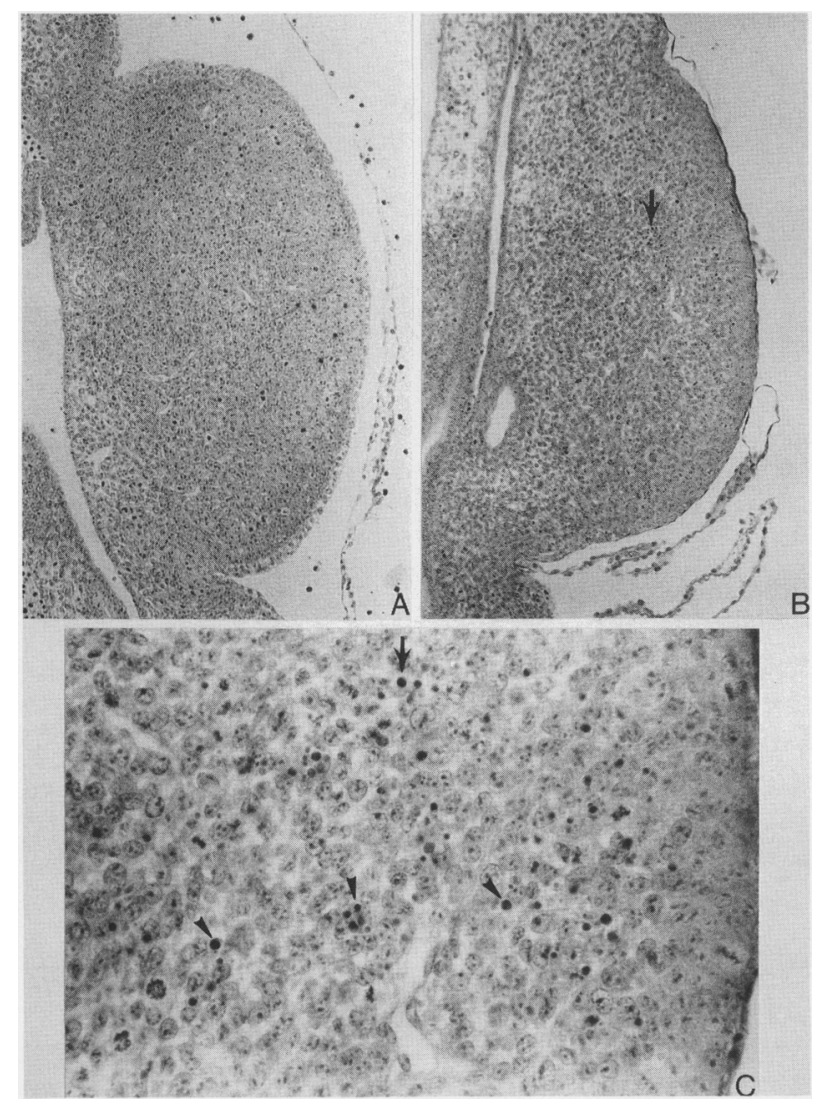

Figure 3. Histological analysis of hindlimb buds. $(A \mid$ Tangential section of hindlimb bud (magnification, $(18 \times)$ from wildtype embryo on midday 10 (36 somites). Note thickening of ectoderm at anterior and posterior poles indicative of AER. $(B)$ Tangential section of hindlimb bud (magnification, $18 \times$ ) from $1 g 1 / 1 g 1$ embryo on midday 10 (38 somites). No thickening of the ectoderm is evident. The arrow indicates dead cells in the anterior central mesoderm. $(C)$ Enlargement of B (magnification, $45 \times$ / with arrow indicating same dead cell. Arrowheads denote other dead cells. thickening was found, but most of the cells therein were necrotic.

Another feature of mutant hindlimb buds was the absence of a marginal venous sinus (Fig. 4). This channel, which normally underlies the AER, was first seen in wild-type embryos of the 30- to 35-somite stage but could not be found in any mutant hindlimb bud.

\section{Retinoic acid hypersensitivity}

Retinoic acid (RA) has been shown to alter the phenotypes of certain mutant mice, including curly tail (Seller et al. 1979) and Splotch (Kapron-Bras and Trasler 1984). Therefore, we decided to search for any interaction between RA and lgl. Administration of $25 \mathrm{mg} / \mathrm{kg}$ of RA intraperitoneally on day 9.5 of gestation ( $\sim 25$-somite stage) to six $1 g 1 /$ + dams yielded 42 viable day 18 fetuses. Of these, 13 fetuses were not transgenic (wild type), 21 were hemizygous for the pHT1 insertion, and 8 were homozygous and displayed the mutant phenotype. The severity of the $I g l$ phenotype in comparison to untreated fetuses (McNeish et al. 1990) was significantly increased, as can be seen in Table 1. The results indicate that both forelimbs and hindlimbs of $l g 1 / 1 g l$ fetuses are missing more skeletal elements than $l g l /+$ or $+1+$ fetuses exposed to RA during the period of early limb development. The forelimb phenotype remains more severe preaxially or anteriorly as evidenced by $14 / 16$ missing radii versus only 4/16 missing ulnae. Craniofacial clefting, a frequent feature of the $1 g l / 1 g l$ offspring, was evident in all but one RA-exposed $1 g l / l g l$ fetus. At this dose of RA, a low frequency of cleft palate was induced in $+1+$ and $1 g l /+$ fetuses as well. Malformations of the tail were rarely encountered in untreated $l g l / l g l$ fetuses $(\mathrm{McNeish}$ et al. 1990|, yet 4 of $81 g 1 / / g l$ fetuses were completely missing the tail, with only 2 of 34 wild-type and hemizygous mice showing this effect.

\section{Genetic background effects on the lgl phenotype}

The $1 g 1$ mutation was produced in C57BL6/C3H hybrid mice. We were interested in determining whether the phenotype of homozygous mutants would vary when expressed on a different genetic background. To this end, lgl hemizygous females were crossed to wild-type males of the inbred BALB/c strain. BALB/c was chosen because it is more distantly related to $\mathrm{C} 57 \mathrm{BL} / 6$ and $\mathrm{C} 3 \mathrm{H}$ than many other common laboratory strains (Hogan et al. 1986). Transgenic female offspring were backcrossed to wild-type BALB/c males, resulting in successive generations with increasing proportions of their genomes contributed by the BALB/c strain.

After six generations the genetic background is $\sim 98.4 \% \mathrm{BALB} / \mathrm{c}$. Hemizygous $\mathrm{lgl}$ males and females were then mated to produce homozygous mutants, which exhibited brain and forelimb defects indistinguishable from those observed previously on the C57BL6/C3H background. The hindlimb defects, however, were much less severe in the BALB/c strain. The mutants had significant hindlimb development distal to 
Singh et al.

Figure 4. Cell death in mutant hindlimb buds. (A) Tangential section of hindlimb bud from late day 10 wild-type embryo (40 somites). Note thickened ectoderm covering much of the anterior half of the limb bud and the underlying marginal venous sinus (arrowheads). Magnification, $21.2 \times$. (B) Tangential section of hindlimb bud from $1 g 1 / \mathrm{lg} 1$ embryo late on day $10 / 40$ somites). No evidence of AER or marginal venous sinus. Magnification, $21.2 \times .(C)$ Enlargement (magnification, $53 \times$ ) of $B$ displaying the extent of cell death in the hindlimb bud mesoderm and ectoderm. Note extensive cell death in ectoderm /small arrowhead) and distal mesoderm (large arrowhead). Arrows indicate mitotic figures indicating cell division.

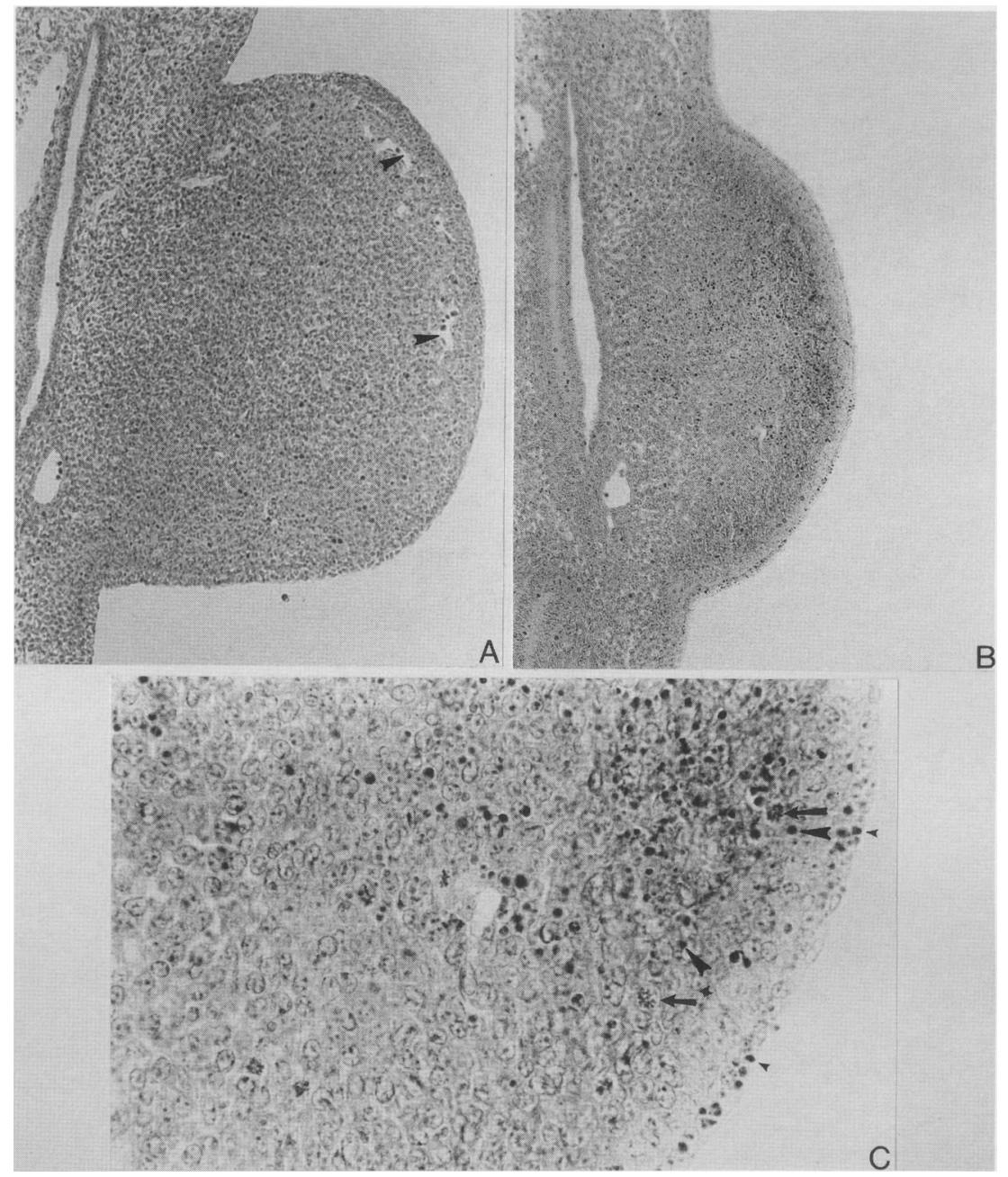

both transgene and nontransgene DNA. A number of conventional cloning strategies failed, apparently because of the extreme hypermethylated state of the transgene and the restriction systems present in most Escherichia coli host strains (data not shown). Nevertheless, two approaches succeeded eventually in generating junction segment clones. First, Southern blots of StuI-digested, partially purified transgene concatemer DNA

Table 1. Expression of selected malformations in offspring of mice treated with all-trans retinoic acid

\begin{tabular}{|c|c|c|c|c|}
\hline \multirow[b]{3}{*}{ Malformation } & \multirow{3}{*}{$\frac{\lg l / l g I^{\mathbf{a}}}{\%(n)}$} & \multicolumn{3}{|c|}{ Retinoic acid $(25 \mathrm{mg} / \mathrm{kg})$} \\
\hline & & $\lg 1 / \operatorname{lgl}$ & $1 g 1 /+$ & $+1+$ \\
\hline & & $\%(n)$ & $\%(n)$ & $\%(n)$ \\
\hline Missing femur & $2(88)$ & $25(16)$ & $0(42)$ & $0(26)$ \\
\hline Missing or rudimentary humerus & $5(88)$ & $25(16)$ & $0(42)$ & $0(26)$ \\
\hline Missing radius & $44(88)$ & $88(16)$ & $0(42)$ & $0(26)$ \\
\hline Missing ulna & $2(88)$ & $25(16)$ & $0(42)$ & $0(26)$ \\
\hline Craniofacial cleft & $51(130)$ & $88(8)$ & $5(21)$ & $8(13)$ \\
\hline Tailless & $0\lfloor 130\}$ & $50(8)$ & $10(21)$ & $0(13)$ \\
\hline
\end{tabular}

Retinoic acid was administered intraperitoneally on day 9.5 of gestation.

${ }^{a}$ Results are from McNeish et al. (1990). 


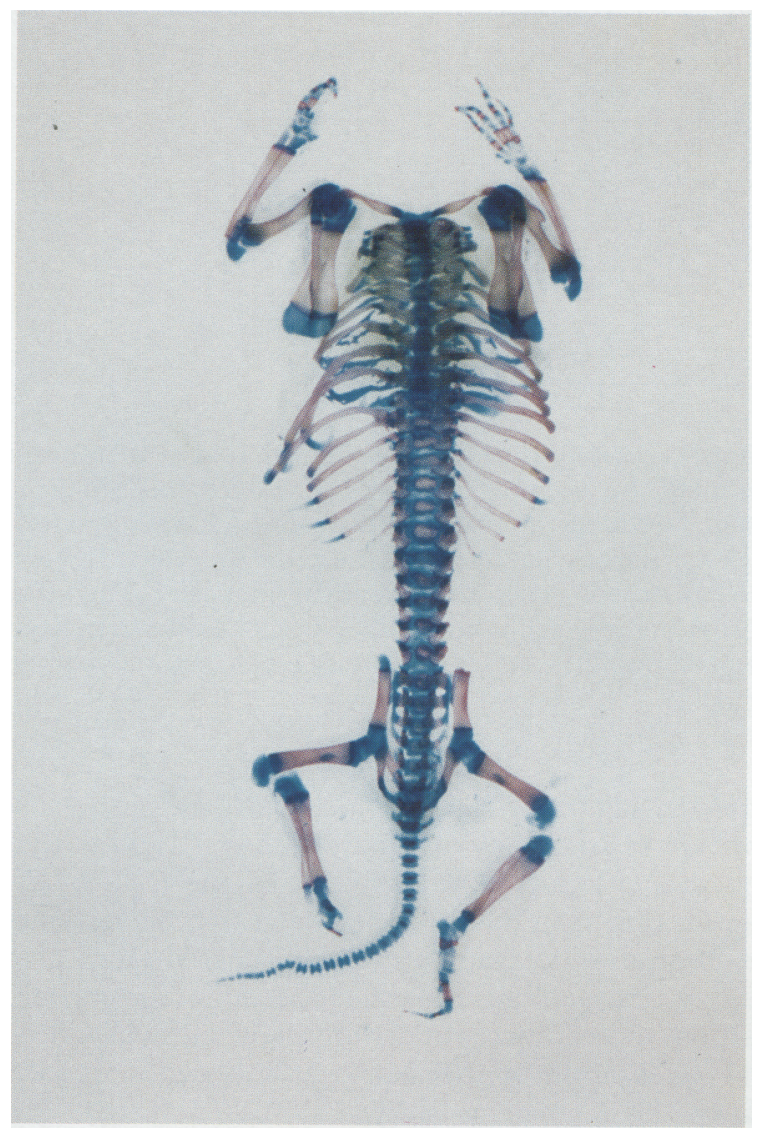

Figure 5. Skeletal preparation of a newborn $1 g 1 / / g 1$ mouse pup after breeding for six generations onto the BALB/c background. Note extensive hindlimb development distal to the femur.

gave a single very intense band of hybridization to a transgene probe and three faint bands, presumably representing single copy junction segments (for details, see Materials and methods). To clone these putative junction segments, DNA was purified from appropriate gel regions, ligated to known sequences (pBS plasmid), and polymerase chain reaction (PCR)-amplified to eliminate methylation. The product segments were then purified and used to generate plasmid libraries that were screened with a transgene probe. All three StuI segments isolated in this manner carried true junctions between pHT1 transgene and non-pHT1 DNA. Only one, however, carried mouse DNA. We were surprised to learn that the other two junctions were between pHT1 transgene and E. coli genomic DNA, which had apparently contaminated the original preparation of pHT1 DNA microinjected into zygotes and was consequently incorporated into the transgene concatemer. The identities of the nonpHT1 portions of the junction segments were determined by using them as probes to hybridize to mouse (transgenic and nontransgenic) and $E$. coli genomic DNA Southern blots (data not shown).

A second successful cloning approach used plasmid rescue in the extremely restriction-deficient $\mathrm{DH} 10 \mathrm{~B}$ cells (Grant et al. 1990). Genomic DNA from $1 g 1 / 1 g 1$ mice was partially digested with Sau3AI, and segments $>12$ $\mathrm{kb}$ in size were selected from an agarose gel. This DNA was circularized by ligation under dilute conditions $\mid<1$ $\mu \mathrm{g} / \mathrm{ml}$ ) and used to transform DH10B cells, which were grown on ampicillin plates. We observed that thousands of rescue clones were obtained readily with these cells, although much more exhaustive plasmid rescue efforts with other strains of $E$. coli had previously failed to yield any.

The original pHTl transgene construct used to make Igl mice had been linearized by cleavage at the ScaI site within the ampicillin-resistance gene of pBR322. Therefore, successful plasmid rescue of transgene sequences required the exact reconstruction of this gene during the generation of the transgene concatemer. Genomic Southern blot experiments indicated that $>90 \%$ of the ScaI sites had been recreated in the transgene. Nevertheless, some portions of the transgene may remain "unrescuable" as a result of an inactive ampicillin-resistance gene resulting from base changes during rejoining or occasional head-to-head or tail-to-tail ligations.

We first screened the rescue clones with a total mouse genomic DNA probe at reduced stringency to identify clones carrying mouse repeat DNA sequences. Nine independent overlapping clones representing a single junction, designated $\mathrm{Jl}$, were identified. Although this single junction was rescue-cloned nine times, the repeat probe screening did not appear to retrieve all junctions, as the previously PCR-cloned mouse-transgene junction was missed. To characterize thoroughly the transgene insertion, we therefore conducted an exhaustive search for sequences flanking the transgene by preparing DNA from $\sim 1000$ individual rescue clones. These clones were then screened for the presence of nontransgene DNA sequences by restriction digestion with BstXI and BstEII, which do not cleave within the pHT1 transgene. Any DNAs cut by these enzymes were analyzed further by hybridization to mouse and E. coli DNA to determine the nature of the nontransgene sequences. The clones were then restriction mapped to place them in overlapping groups representing individual junctions.

This analysis yielded five additional overlapping clones of the J1 transgene-mouse DNA junction rescued previously and 16 clones of the transgene-mouse junction isolated already by PCR, designated J2. It also produced six clones of one $E$. coli-transgene junction and 10 clones of the other $E$. coli-transgene junction isolated previously by PCR. Furthermore, we found two additional transgene $-E$. coli junctions, obtaining five clones of one and four clones of the other. The isolation of a total of four distinct junctions with $E$. coli DNA suggested the presence of at least two blocks of $E$. coli genomic DNA inserted within the transgene concatemer.

The 11 mouse sequences were then used to screen a wild-type mouse genomic cosmid library, resulting in the identification of two cosmids, C-10 and C-11 that, together, spanned $60 \mathrm{~kb}$ surrounding this transgene insertion site (Fig. 6). These were restriction mapped, and a 


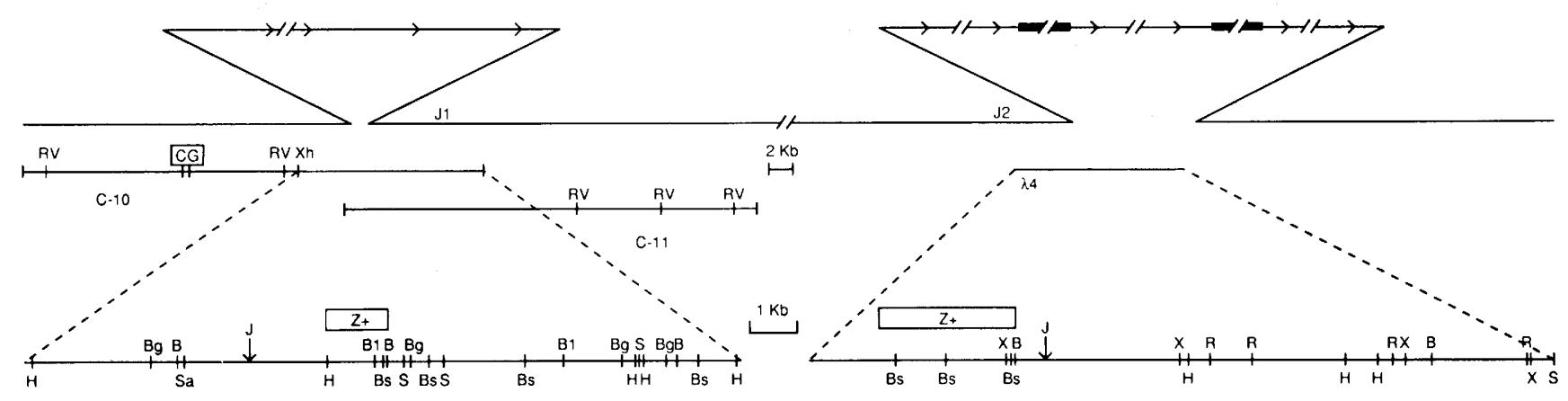

Figure 6. Model of the transgene disruption. The horizontal arrows at the top represent the two tightly linked transgene insertions. The solid rectangles are E. coli genomic DNA sequences integrated into one of the transgene concatemers. (J1 and J2) Mouse sequences found at transgene junctions. The gaps in the mouse sequence line denote the deletions generated by the transgene insertions. (C-10 and C-11) Cosmid clones of wild-type DNA that span the J1 region; $(\lambda 4)$ A $\lambda$ clone of wild-type DNA from the J2 region. The boxed CG marks the position of a CG island; the boxed $Z$ + sequences were found to be highly conserved phylogenetically. The vertical arrows labeled J indicate where transgene-mouse DNA junctions were located on the J1 and J2 plasmid rescue clones. Restriction sites indicated are BamHI (B), EcoRV (RV), HindIII (H), XhoI (Xh), SalI (Sa), BstEII (Bs), StuI (S), BglI (Bg), BglII (BI), XbaI (X), and EcoRI (R).

number of restriction segments from around the junction region were used to probe genomic Southern blots of $l g l / l g l, l g l /+$, and $+/+$ DNA. The results confirmed that this was a true junction and indicated that the transgene insertion at this site was associated with an $\sim 1-\mathrm{kb}$ DNA deletion (data not shown).

Interestingly, the J2 mouse flanking DNA sequences, originally isolated by PCR, were not present on either the $\mathrm{C}-10$ or $\mathrm{C}-11$ cosmid clone, suggesting the presence of two distinct transgene insertions that are separated by $\geqslant 30 \mathrm{~kb}$ of chromosomal DNA. Nevertheless, our Southern analyses of hundreds of progeny of $1 g l /+$ matings and chromosomal mapping studies described later indicate that the two loci are tightly linked. The J2 mouse sequences were used to retrieve a wild-type mouse DNA $\lambda$ clone $(\lambda 4)$ that covered this junction region. Genomic Southern blots, with various restriction segments from this clone as probes, again confirmed that this was a true junction region and showed that this transgene insertion resulted in a deletion of $\geqslant 10 \mathrm{~kb}$ of mouse DNA /data not shown).

\section{Field inversion gel electrophoretic analysis}

To better understand the nature of the transgene disruption, we digested $l g l / l g l$ DNA with restriction enzymes that did not cut within the pHT1 sequences of the transgene (BstEII or BstXI). This DNA was resolved by field inversion gel electrophoresis (FIGE), Southern blotted, and hybridized to $\mathrm{pHTl}$ transgene sequence probe. As shown in Figure 7, four distinct bands of hybridization were observed, suggesting the presence of four separate blocks of pHT1 transgene DNA. A number of control experiments with varying amounts of restriction enzyme and different lengths of digestion demonstrated that these bands were not the result of incomplete cleavage (data not shown). The sizes of these transgene blocks were estimated to be $480,310,190$, and $55 \mathrm{~kb}$.

The simplest model compatible with the data is pre- sented in Figure 6. In this model two transgene insertions are separated by at least $30 \mathrm{~kb}$ of DNA, as the J2 sequences are not found on either of the C-10 or C-11 cosmids that span the other insertion site. Nevertheless, the chromosomal mapping data (discussed later) places the two transgene insertions at the same chromosomal position, indicating that they are tightly linked. The relative orientation of the DNAs at the two sites is unknown. FIGE analysis suggested that BstEII or BstXI cleave the mouse DNA flanking the transgene insertions, as well as the two blocks of E. coli DNA within the $\mathrm{J} 2$ insertion, producing the observed four segments of

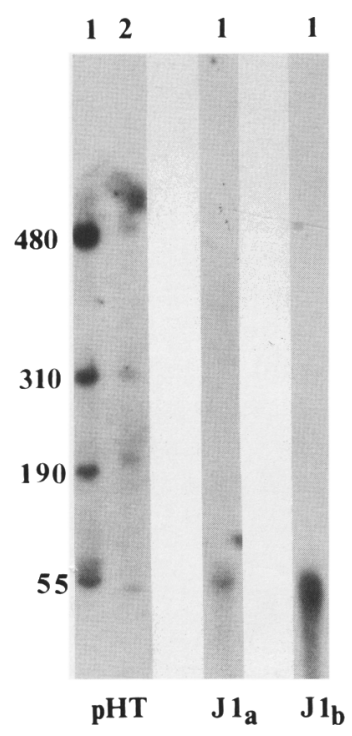

Figure 7. Blot hybridization of $l g l / l g l$ genomic DNA digested with BstEII (1) and BstXI (2). Genomic DNA digests were resolved by FIGE (for details, see Materials and methods), blotted, and hybridized with ${ }^{32} \mathrm{P}$-radiolabeled transgene DNA (pHT) and sequences flanking either end of $\mathrm{J} 1$ junction $(\mathrm{Jl} \mathrm{a}, \mathrm{J} 1 \mathrm{~b})$. The sizes of the hybridizing bands are in kilobase pairs. 
pHT1-containing DNA. The smallest segment, of $\sim 55$ $\mathrm{kb}$, hybridizes to mouse flanking DNA from both sides of the Jl transgene insertion, indicating that it does not carry either of the two blocks of E. coli genomic DNA. The smaller transgene insertion resulted in a deletion of $\sim 1 \mathrm{~kb}$, whereas all $10 \mathrm{~kb}$ of the mouse DNA of the $\lambda 4$ clone beyond the $\mathrm{J} 2$ junction site is absent in $1 g 1 / 1 g 1$ homozygotes, thus establishing a lower limit for the size of this deletion. Of the eight predicted junction segments between pHT1 transgene DNA and non-pHT1 DNA, six have been isolated, but two appear to be particularly difficult to clone by plasmid rescue.

\section{Chromosome mapping}

The chromosomal positions of the two transgene insertions were determined by hybridizing single-copy mouse sequences from the $\mathrm{J} 1$ and $\mathrm{J} 2$ junction clones to an interspecific backcross panel derived by crossing C57BL/6J and Mus spretus. This panel has been typed for $>800$ loci that are well distributed among all of the autosomes as well as the X chromosome (Copeland and Jenkins 1991). DNAs from C57BL/6J and Mus spretus were digested with several restriction enzymes and analyzed by Southern blot hybridization to identify informative restriction fragment length polymorphisms (RFLPs) with each of the transgene flanking probes. The strain distribution pattern of each RFLP in the interspecific backcross was then used to determine the chromosomal position of the transgene insertions.

The transgene insertions were localized to the distal region of chromosome 12 . N2 progeny (162) were typed for the immunoglobulin heavy-chain gene $(\operatorname{IgH})$ locus and a single-copy restriction segment from the J1 region, with no recombinants observed. This places the transgene insertion within $1.8 \mathrm{cM}$ of the $I g H$ locus, within $95 \%$ confidence limit. This chromosomal location was confirmed independently by in situ hybridization of biotinylated transgene probe (pHT1) to metaphase chromosomes isolated from $1 g 1 / 1 g 1$ liver cells (J. Crolla and N. Brown, pers. comm.). Interspecific backcross mapping of the $\mathrm{J} 2$ region placed it at the same position as J1. Significantly, these transgene insertions also mapped, within experimental error, at the same location as the iv gene (Brueckner et al. 1989; Hanzlik et al. 1990). This is of particular interest as the homozygous iv/iv mice display a phenotype remarkably similar to $l g l / l g l$ mice, with $50 \%$ having situs inversus, a mirror image reversal of internal organ symmetry. The iv/iv mice, however, have normal limb, craniofacial, and brain morphology.

\section{$\operatorname{lgl}$ is allelic with iv}

To test for possible allelism, we mated iv/iv homozygotes of the SI/Col strain (The Jackson Laboratory, Bar Harbor, ME) with $l g l /+$ hemizygotes. Among 30 progeny, all of which carried an iv allele, 15 were found by Southern analysis to have the $1 g l$ transgene insertion; among these, $10(67 \%)$ showed situs inversus as determined by the position of the milk spot or visceral exam- ination when necessary. These results clearly showed that the iv and $\mid g l$ mutations do not complement each other with respect to the inverted viscera phenotype; therefore, the two loci are allelic. It should be noted, however, that none of the progeny mice had any of the other malformations associated with the $1 g 1$ phenotype. This suggests that the transgene insertion disrupted one gene allelic with iv and other gene(s) important in limb, craniofacial, and brain development.

\section{Candidate genes}

The C-10 and C-11 cosmid clones carrying $\sim 60 \mathrm{~kb}$ of DNA surrounding the J1 transgene insertion were restriction mapped (Fig. 6). This analysis revealed the presence of a very pronounced CG or HpaII tiny fragment (HTF) island $\sim 14 \mathrm{~kb}$ from the insertion. Within a $1-\mathrm{kb}$ region were located one NruI, one NaeI, two SacII, and two BssHII recognition sites. These 6-bp sequences are all rich in guanosines and cytosines, with three of them (NaeI, SacII, and BssHII) consisting of only Cs and Gs and with NruI having four of six bases of C or G. Furthermore, three of these recognition sites (NruI, BssHII, and SacII) have two CG dinucleotides, which are severalfold under-represented in mammalian DNA. The NaeI site also has one CG. The net result of their base composition and sequence characteristics is that these recognition sites are found quite rarely in mammalian DNA. The presence of a cluster of six of these sequences within a $1-\mathrm{kb}$ region is therefore striking and strongly suggests the presence of a CG island which, in turn, indicates the presence of a gene, as such islands are often found near the $5^{\prime}$ ends of mammalian genes (Bird et al. 1985).

To search for possible coding sequences, we tested restriction segments from the C-10 and C-11 cosmids for evolutionary conservation by hybridizing them at reduced stringency to genomic Southern blots of DNAs from mouse, rat, pig, chicken, human, and Drosophila. A $1.1-\mathrm{kb}$ BamHI-HindIII segment $\sim 3 \mathrm{~kb}$ from the transgene insertion site was found to be particularly well conserved. As shown in Figure 8A, a 750-bp HaeIII subfragment of this segment gave multiple distinct bands of hybridization for all DNAs tested, including Drosophila. The hybridization to several distinct segments in each DNA tested suggested that this coding region might represent a member of a multigene family, and this was confirmed by isolating a number of homologous clones from an 8.5-day C57BL/6J embryonic cDNA library. Initial sequence analysis of these cDNAs demonstrates that they are derived from distinct yet closely related genes (data not shown).

Single-copy mouse DNA segments from the J2 insertion region were also used as probes in "zoo" blots to identify possible coding sequences. As shown in Figure $8 \mathrm{~B}$, there is evidence for considerable evolutionary conservation of the $2.9-\mathrm{kb} B g I I I-B a m H I$ restriction segment, which cross-hybridizes at $58^{\circ} \mathrm{C}$ to rat, human, pig, chicken, and even Drosophila genomic DNA. At higher stringency $\left(65^{\circ} \mathrm{C}\right)$, discrete hybridization bands are re- 


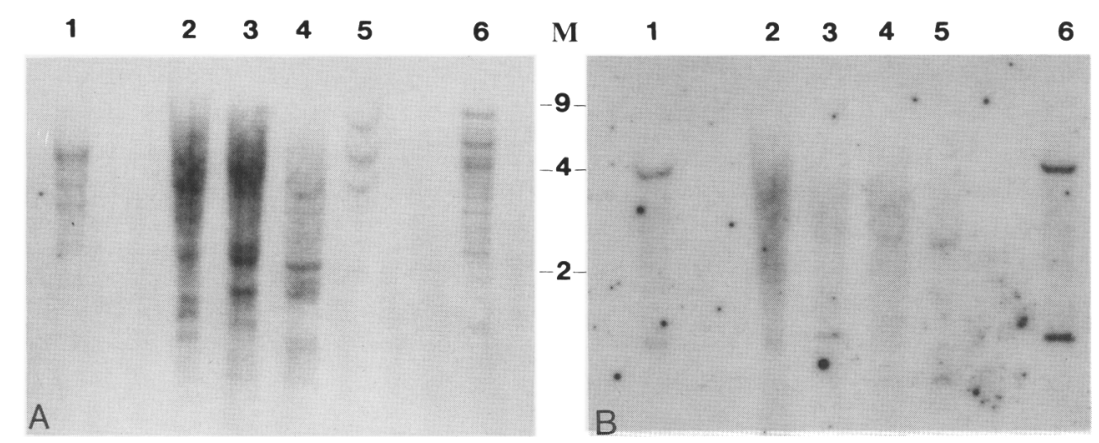

Figure 8. Southern blot analysis demonstrating evolutionary conservation of sequences flanking the J1 and J2 junctions. PvuIIdigested genomic DNAs from rat (lane 1), human (lane 2), pig (lane 3), chicken (lane 4), Drosophila (lane 5), and wild-type mouse (lane 6) were hybridized $\left(58^{\circ} \mathrm{C}\right)$ with a 750 -bp HaeIII subfragment from the well-conserved 1.1-kb HindIII-BamHI restriction segment near the $\mathrm{Jl}$ junction $(A)$ and the $2.9-\mathrm{kb} B g l \mathrm{II}-\mathrm{BamHI}$ fragment from the $\mathrm{J} 2$ junction $(B)$. The amount of genomic DNA digested was $5 \mu \mathrm{g}(\mathrm{rat})$, $10 \mu \mathrm{g}$ (human), $15 \mu \mathrm{g}$ (pig), $15 \mu \mathrm{g}$ (chicken), $15 \mu \mathrm{g}$ (Drosophila), and $8 \mu \mathrm{g}$ (mouse). Size markers are in kilobase pairs (M).

tained in rat, chicken, and human DNA, further suggesting phylogenetic conservation of $\mathrm{J} 2$ sequences (data not shown). This segment was then used to screen an 11.5 day mouse embryonic cDNA library, yielding several clones that are currently being investigated further.

\section{Discussion}

The $l g 1$ insertional mutation has apparently disrupted genes involved in some interesting facets of mammalian development. Limb morphogenesis has long provided an important model to understand pattern formation during vertebrate development with many classic experimental approaches shedding light on the function of developing limb regions, including the AER, the mesenchymal progress zone, and the ZPA. Furthermore, more contemporary experiments have revealed interesting expression patterns in developing limbs for a variety of genes believed to be developmentally important (Brockes 1989). The $1 g l$ mutation promises to help combine the molecular, embryologic, and genetic approaches by providing molecular access to genes that clearly play a significant role in limb morphogenesis. In addition to limb defects, the $1 g 1 / 1 g 1$ mice show craniofacial malformations and abnormal development of the anterior parts of the brain, and $50 \%$ of them have situs inversus, a mirror image arrangement of internal organs.

Histologic and SEM analysis of developing $1 g 1 / 1 g l$ hindlimbs revealed several interesting abnormalities. Early limb bud formation during days 9 and 10 appears normal when viewed grossly with regard to growth and form. But by day 11 the mutant hindlimbs are smaller in size and misshapen. Closer inspection, however, reveals that day $101 g l / l g l$ hindlimb buds lack an AER, as well as the underlying venous sinus and have an unusually large number of dying cells in the mesoderm and ectoderm. Thus, the deficiency leading to the $l g l$ hindlimb phenotype has its effect during early day 10 around the stage of 30-35 somites. The mesenchymal cells, which originally migrate from the lateral plate mesoderm to initiate limb bud formation, have a key role in limb development
(Harrison 1918). Removal of these mesenchymal cells blocks limb formation, whereas grafting of mesenchymal cells can generate a limb at a new position. Furthermore, combining hindlimb mesenchyme with forelimb ectoderm results in the formation of a hindlimb, and limb ectoderm grafting does not produce a limb at the new site. Removal of the AER will, however, cause cessation of further limb development (Saunders 1948). It is interesting to note that removal of the AER from an early stage chick wing bud results in a limb with only the humerus and no distal structures (Rowe et al. 1982). In $1 g l / l g l$ mutants the absence of limb skeleton distal to the femur suggests an early loss of AER function and coincides with our morphologic observations that did not demonstrate any typical AER throughout the period of hindlimb bud formation. Moreover, the pattern of cell death in the $1 g l / l g l$ hindlimb bud is similar to that reported in chick limb buds with AER removed (Rowe et al. 1982). These workers describe cell debris first appearing in the anterior mesoderm, followed by an extension to include most of the distal mesoderm across the anteroposterior axis of the limb. Consistent with the lack of AER is the absence of a marginal venous sinus in mutant hindlimbs. Feinberg and Saunders (1982) have shown that AER is required for the development of these vessels in the chick; thus, the absence of this vascular channel in mutant hindlimbs further strengthens the conclusion that AER function is absent in $\mid g l / l g l$ hindlimbs.

It is interesting to note that the many teratogenic effects of RA treatment include missing olfactory bulb, cleft palate, situs inversus, and malformed limbs (Shenefelt 1972). In this report we studied the developmental effects of low levels of RA, which result in very few malformations in wild-type and hemizygous $1 g 1$ mice. The observed hypersensitivity of the $1 g 1 / 1 g 1 \mathrm{mu}-$ tant embryos to exogenous RA provides intriguing hints concerning the possible molecular pathways disrupted by the transgene insertion. The increased severity of the resulting phenotype, sometimes producing a near-complete absence of all limbs and tail, indicates a reduced 
ability to contend with artificially elevated RA levels. This, in turn, suggests a possible alteration in RA-sensing or-degradation pathways, although even this rather general proposition remains quite speculative.

The observed genetic background effects may be explained by the actions of other strain-specific genes. It is possible that other genes exist whose functions overlap those of $1 g 1$; alleles of these genes specific to $\mathrm{BALB} / \mathrm{c}$ may partially compensate for the loss of $l g l$ function, allowing mutant hindlimb development to progress further than in $\mathrm{C} 57 \mathrm{BL} / \mathrm{C} 3 \mathrm{H}$ mice. Alternatively, the genes mutated in $1 g 1 / 1 \mathrm{gl}$ mice may not be inactivated by a physical disruption but, rather, by an epigenetic modification such as hypermethylation. Our early attempts at cloning the DNA flanking the transgene insertion revealed that the transgene sequences had become extremely hypermethylated in the $\mathrm{C} 57 \mathrm{BL} / \mathrm{C} 3 \mathrm{H}$ mice (data not shown). We suspected that the genes responsible for the $l g l$ mutant hindlimb phenotype were inactivated by methylation owing to their proximity to transgene sequences that direct methylation. Strain-specific modifier genes are known to exist that can control patterns of transgene methylation (Engler et al. 1991). However, isochizomer analysis with the restriction enzymes HpaII and MspI demonstrated that the methylation status of the transgene was unchanged on breeding to the $\mathrm{BALB} / \mathrm{c}$ background (data not shown).

Molecular analysis of the transgene disruption demonstrated the presence of two distinct transgene insertions separated by at least $30 \mathrm{~kb}$ of DNA. The probability of two independent transgene insertions occurring within $0.5 \mathrm{cM}$ of each other, as was observed in $1 g l$ mice, is extremely low. This suggests that the two events were linked, perhaps with an initial large concatemer undergoing simultaneous multiple illegitimate recombinations with this chromosomal region, resulting in the double insertion with a residual "island" of mouse genomic DNA between. Chromosomal mapping studies with mouse single-copy DNA sequences flanking each of these insertions showed the two transgene blocks to be linked very closely and to reside at or near the iv locus. Furthermore, complementation tests demonstrated allelism between the $\lg 1$ and iv mutations. Interestingly, however, the $1 g 1 /$ iv mice showed situs inversus but no limb, brain, or craniofacial abnormalities, suggesting that the transgene insertion disrupted more than one gene. Alternatively, the $l g l$ mutation might simply represent a more severe or null allele of the iv gene.

The $1 g 1$ insertional mutation promises molecular access to a gene of key importance in the establishment of bilateral asymmetry. The process by which the developing embryo distinguishes left from right to generate asymmetries of organ formation and location is poorly understood. It is known, however, that $\sim 50 \%$ of the mice homozygous for the autosomal recessive iv gene exhibit reversed left-right asymmetry, as if the process were randomized (Hummel and Chapman 1959). Interestingly, a careful analysis shows that in $\sim 40 \%$ of these iv/iv mice one observes only partial reversal (Layton 1976). Moreover, in $\sim 20 \%$ of iv/iv mice heart defects result from a disarrayed process of cardiac looping (Layton 1978|, probably caused by uncertain establishment of visceral asymmetry.

Similarly, in humans many heart defects have been associated with situs inversus or situs ambiguous, an incomplete visceral inversion (Arnold et al. 1983). The polysplenia, asplenia, and Kartegener "syndromes" are closely related genetically, with reported sibships in which one sibling has polysplenia and the other has asplenia (Polheus and Schafer 1954; Zlotogora and Elian 1981) or in which one sibling has polysplenia and the other has Kartegener syndrome (Schidlow et al. 1982). Each condition represents a developmental defect in asymmetry, with polysplenia, for example, associated with bilateral left-sidedness with multiple spleens and frequently two left lungs, and congenital cardiac defects including atrial and ventricular septal defects, right aortic arch, endocardial cushion defects, pulmonic stenosis, double outlet right ventricle, and patent ductus arteriosus. Asplenia is a bilateral right-sidedness, and Kartegener syndrome is a situs inversus condition. Proteoglycan synthesis (Yost 1990) and adrenergic mechanisms (Fujinaga and Baden 1991) have been implicated in situs determination, and interesting theoretical models have been proposed (Brown and Wolpert 1990); nevertheless, the molecular mechanisms remain obscure. The molecular handle provided by the $l g l$ transgene insertion should greatly facilitate identification of the iv gene.

We are in the initial stages of characterizing two candidate genes found flanking the two transgene insertions. Analysis of the structures of these genes in wildtype, $I g l$, and iv mice might provide clues concerning their identities. Definitive proof of their biological function, however, will require transgene rescue and/or independent individual disruption by homologous recombination with embryonic stem cells.

\section{Materials and methods}

\section{Animals and embryos}

Mice used in these studies were from the pHTl-1 line inbred on a C57BL6/C3H hybrid background for many generations. Heterozygous offspring were identified by Southern blot hybridization and autoradiography and mated overnight to produce timed pregnancies. The morning of finding a vaginal plug was considered day 0.

\section{Histology and SEM}

On day 10 or 11 of gestation, mice were sacrificed, by cervical dislocation and the embryos with their extraembryonic membranes were removed from the uterus. Under a dissecting microscope, the yolk sac was removed and frozen for subsequent genotype identification by Southern blotting. Somite number of each embryo was determined, and the embryo was then fixed in Bouin's fluid for routine histology or in 3\% glutaraldehyde, 3\% paraformaldehyde, in cacodylate buffer for SEM. These latter embryos were dehydrated in alcohol and by critical-point drying. Surface coating with gold-palladium was carried out with an SEM coating unit (5100 Polaron). Pictures were taken on an ISI-100 scanning electron microscope. Embryos for routine his- 
tology were embedded to cut $6-\mu \mathrm{m}$ tangential sections of hindlimb buds, which were stained with hematoxylin and eosin.

\section{$R A$}

On day 9.5 of pregnancy, six mice were given all-trans RA (Sigma) by intraperitoneal injection. Under conditions of yellow light, RA was dissolved in a small quantity of corn oil and given at a dose of $25 \mathrm{mg} / \mathrm{kg}(10 \mathrm{ml} / \mathrm{kg}$ of $2.5 \mathrm{mg} / \mathrm{ml}$ solution). These dams were sacrificed on day 18 , and the fetuses were examined for external malformations and fixed in $95 \%$ ethanol for skeletal analysis by alizarin red and alcian blue staining (Kuczuk and Scott 1984).

\section{PCR cloning of junctions}

The transgene sequences were purified partially by digestion of homozygous $1 g l / l g 1$ DNA with BstEII and BstXI, which do not cleave within the $\mathrm{pHTl}$ transgene concatemer, electrophoresis on a $0.6 \%$ agarose gel, and electroelution of DNA segments $>25$ $\mathrm{kb}$ in size. This DNA was cut with a variety of restriction enzymes, electrophoresed, blotted, and probed with five distinct segments of pHTl to search for faint bands representing possible junction segments. Cleavage with StuI, followed by hybridization to a 876-bp AatII segment of pHT1, gave a single intense band of $8.8 \mathrm{~kb}$, the size of the unit repeated in the transgene concatamer, and three faint bands of $2.3,1.6$, and $1.3 \mathrm{~kb}$. These putative junctions were excised from appropriate gel regions, ligated to SmaI-cut pBS to give known end sequences, and PCRamplified. These amplified segments were then used to generate pBS libraries, from which the three $\mathrm{pHTl}$-non-pHTl junction segments were identified.

\section{High-molecular-weight DNA preparation and FIGE}

Genomic DNA from $1 g l / l g 1$ mice was prepared in agarose inserts. Newborn pups were sacrificed and the tissue was macerated with autoclaved glass coverslips and ice-cold PBS (Whittaker). The intact cell suspension was warmed at $35-40^{\circ} \mathrm{C}$ and mixed with an equal volume of low-gelling $1 \%$ InCert agarose (FMC) in $0.1 \mathrm{~mm}$ EDTA, $10 \mathrm{~mm}$ Tris- $\mathrm{HCl}(\mathrm{pH} 8.0)$, at $55^{\circ} \mathrm{C}$. The cell suspension-agarose slurry was poured quickly into moulds (Pharmacia-LKB) and cooled at $4^{\circ} \mathrm{C}$ for $15 \mathrm{~min}$. The gel inserts were processed for genomic DNA preparation and subsequent restriction cleavage according to Smith et al. (1986).

The restriction digests were resolved by FIGE (Carle et al. 1986; Chen et al. 1988) in $0.5 \times$ Tris borate buffer at $12-16^{\circ} \mathrm{C}$ with a pulse module (DNAstar). A potential difference of 6-8 $\mathrm{V} / \mathrm{cm}$ was maintained throughout the $24-$ to $36-\mathrm{hr}$ electrophoresis run. The initial forward and backward pulses were for 3 and $1 \mathrm{sec}$, respectively, and final forward and backward pulses were for 60 and $20 \mathrm{sec}$, respectively.

\section{Chromosome mapping}

Interspecific backcross progeny were generated by mating $\mathrm{C} 57 \mathrm{BL} / 6 \mathrm{~J} \times \mathrm{M}$. spretus $\mathrm{F}_{1}$ females and C57BL/6) males (Copeland and Jenkins 1991). A total of $162 \mathrm{~N}_{2}$ progeny were typed for the $I g H$ locus and J1- and J2-flanking sequences. Recombinant distances were calculated (Green 1981) with the computer program Spretus Madness developed by D. Dave and A. Buchberg (Frederick, MD).

\section{Recombinant DNA methodology}

Standard recombinant DNA techniques, including DNA preparation, and enzymatic manipulations, bacterial transformation, blotting, and hybridization, were performed according to Sambrook et al. (1989).

\section{Acknowledgments}

We thank Hung $\mathrm{Li}$ and $\mathrm{M}$. Todd Valerius for excellent assistance in preparing and analyzing rescue clone DNAs, Debra J. Gilbert for chromosomal mapping, Jan Hagedorn for manuscript preparation, and Dave Collins and John Clancher for illustrations and photography. This work was supported by National Institutes of Health grant HD24517 to S.S.P. and W.J.S. and National Cancer Institute contract N01-CO-74101 with ABL to N.A.J. and N.G.C. D.S. was funded by ES07051. G.S. is a Children's Hospital Medical Center Board of Trustees fellow.

The publication costs of this article were defrayed in part by payment of page charges. This article must therefore be hereby marked "advertisement" in accordance with 18 USC section 1734 solely to indicate this fact.

\section{References}

Arnold, G.L., D. Bixler, and D. Girod. 1983. Probable autosomal recessive inheritance of polysplenia, situs inversus and cardiac defects in an Amish family. Am. J. Med. Genet. 10: 3542.

Bird, A., M. Taggart, M. Fronmer, O. Miller, and D. McLeod. 1985. A fraction of the mouse genome that is derived from islands of nonmethylated, CpG-rich DNA. Cell 40: 91-99.

Brockes, J.P. 1989. Retinoids, homeobox genes, and limb morphogenesis. Neuron 2: 1285-1294.

Brown, N.A. and L. Wolpert. 1990. The development of handedness in left/right asymmetry. Development 109: 1-9.

Brueckner, M., P. D'Eustachio, and A.L. Horwich. 1989. Linkage mapping of a mouse gene, iv, that controls left-right asymmetry of the heart and viscera. Proc. Natl. Acad. Sci. 86: 5035-5038.

Carle, G.F., M. Frank, and M.V. Olson. 1986. Electrophoretic separation of large DNA molecules by periodic inversion of the electric fields. Science 232: 65-68.

Chen, J.D., M.J. Denton, G. Morgan, J.H. Pearn, and A.G. Mackinlay. 1988. The use of field inversion gel electrophoresis for deletion detection in Duchenne muscular dystrophy. Am. I. Hum. Genet. 42: 777-780.

Copeland, N.G. and N.A. Jenkins. 1991. Development and applications of a molecular genetic linkage map of the mouse genome. Trends Genet. 7: 113-118.

Engler, P., D. Haasch, C.A. Pinkert, L. Doglio, M. Glymour, R. Brinster, and U. Storb. 1991. A strain-specific modifier on mouse chromosome 4 controls the methylation of independent transgene loci. Cell 65: 939-947.

Fallon, J.F. and G.M. Crosby. 1977. Polarizing zone activity in limb buds of amniotes. In Vertebrate limb and somite morphogenesis (ed. D.A. Ede, T.R. Hirschliffe, and M. Balls), pp. 55-69. Cambridge University Press, Cambridge, England.

Feinberg, R.N. and J.W. Saunders. 1982. Effects of excising the apical ectodermal ridge on the development of the marginal vasculature of the wing bud in the chick embryo. $/$. Exp. Zool. 219: 345-354.

Fujinoga, M. and J.M. Baden. 1991. Evidence for an adrenergic mechanism in the control of body asymmetry. Dev. Biol. 143: 203-205. 
Grant, S.G.N., J. Jessee, F.R. Bloom, and D. Hanahan. 1990. Differential plasmid rescue from transgenic mouse DNAs into Escherichia coli methylation-restriction mutants. Proc. Natl. Acad. Sci. 87: 4645-4649.

Green, E.L. 1981. Linkage, recombination, and mapping. In $G e-$ netics and probability in animal breeding experiments (ed. M.C. Green), pp. 77-113. Macmillan, New York.

Hanzlik, A.J., M. Binder, W.M. Layton, L. Rowe, M. Layton, B.A. Taylor, M.M. Osemlak, J.E. Richards, D.M. Kurnit, and S.D. Gordon. 1990. The murine situs inversus viscerum (iv) gene responsible for visceral asymmetry is linked tightly to the Igh- $C$ cluster on chromosome 12 . Genomics 7: 389-393.

Harrison, R.G. 1918. Experiments on the development of the forelimb of Amblystoma, a self-differentiating equipotential system. J. Exp. Zool. 25: 413-461.

Heffron, F., C. Rubers, and S. Falkow. 1975. The translocation of a plasmid DNA sequence which mediates ampicillin resistance: Molecular nature and specificity of insertion. Proc. Natl. Acad. Sci. 72: 3623-3627.

Hogan, B., F. Costantini, and E. Lacy. 1986. Manipulating the mouse embryo. Cold Spring Harbor Laboratory, Cold Spring Harbor, New York.

Hummel, K.P. and D.B. Chapman. 1959. Visceral inversion and associated anomalies in the mouse. J. Hered. 50: 9-13.

Hurle, J.M., Y. Ganan, and D. Macias. 1989. Experimental analysis of the in vivo chondrogenic potential of the interdigital mesenchyme of the chick limb bud subjected to local ectodermal removal. Dev. Biol. 132: 368-374.

Kapron-Bras, C.M. and D.G. Trasler. 1984. Gene-teratogen interaction and its morphological basis in retinoic-acid-induced mouse spina bifida. Teratology 30: 143-150.

Kuczuk, M.H. and W.J. Scott. 1984. Potentiation of acetazolamide induced ectrodactyly in SWV and C57BL/6J mice by cadmium sulphate. Teratology 29: 427-435.

Layton, W.M. 1976. Random determination of a developmental process. I. Hered. 67: 336-338.

1978. Heart malformations in mice homozygous for a gene causing situs inversus. In Morphogenesis and malformation of the cardiovascular system (ed. G.C. Rosenquist and D. Bergsmal, pp. 277-293. Alan R. Liss, Inc., New York.

McNeish, J., W. Scott, and S. Potter. 1988. Legless, a mutation in pHTl line transgenic mice. Science 241: 837-839.

McNeish, J.D., J. Thayer, K. Walling, K.K. Sulik, S.S. Potter, and W.J. Scott. 1990. Phenotypic characterization of the transgenic mouse insertional mutation: legless. I. Exp. Zool. 253: 151-162.

Moerman, D.G., G.M. Berrian, and R.H. Waterston. 1986. Molecular cloning of the muscle gene unc-22 in Caenorhabditis elegans by Tc1 transposon tagging. Proc. Natl. Acad. Sci. 83: 2579-2583.

O'Reilly, C., N.S. Shepherd, A. Periera, Z.S. Schwarz-Sommer, I. Bertram, D.S. Robertson, P.A. Peterson, and H. Saedler. 1985. Molecular cloning of the a1 locus of Zea mays using the transposable elements En and Mu1. EMBO J. 4: 877-882.

Polhemus, D.W. and W.B. Schafer. 1954. Congenital absence of the spleen: Syndrome with atrioventricularis and situs inversus. Pediatrics 9: 696-708.

Rubin, G.M., M.G. Kidwell, and P. Bingham. 1982. The molecular basis of P-M hybrid dysgenesis: The nature of induced mutations. Cell 29: 987-994.

Rowe, D.A., J.M. Cairns, and J.F. Fallon. 1982. Spatial and temporal patterns of cell death in limb bud mesoderm after apical ectodermal ridge removal. Dev. Biol. 93: 83-91.

Sambrook, J., E.F. Fritsch, and T. Maniatis. 1989. Molecular cloning: A laboratory manual, 2nd ed. Cold Spring Harbor Laboratory Press, Cold Spring Harbor, New York.
Saunders, J.W. Jr. 1948. The proximo-distal sequence of origin of parts of the chick wing and the role of the ectoderm. J. Exp. Zool. 108: 363-404.

Saunders, J.W. Jr., J.M. Cairns, and M.T. Gasseling. 1957. The role of the apical ridge of ectoderm in the differentiation of the morphological structure and inductive specificity of limb parts of the chick. J. Morphol. 101: 57-88.

Schidlow, D.V., S. Moriberkatz, M.G. Tutz, R.M. Donner, and S. Capasso. 1982. Polysplenia and Kartagener syndrome in two sibships: Association with abnormal respiratory cilia. I. Pediatr. 100: 401-403.

Seller, M.J., S. Embury, P.E. Polani, and M. Adinolfi. 1979. Neural tube defects in curly-tail mice. II. Effects of maternal administration of vitamin A. Proc. R. Soc. Lond. B. Biol. Sci. 206: 95-107.

Shenefelt, R.E. 1972. Morphogenesis of malformations in hamsters caused by retinoic acid: Relation to dose and stage of treatment. Teratology 5: 103-118.

Smith, C.L., P.E. Warkburton, A. Gaal, and C.R. Cantor. 1986. Analysis of genome organization and rearrangement by pulsed filled gradient gel electrophoresis. Genetic Eng. 8: 45-70, Plenum Press, New York.

Summerbell, D. and J.H. Lewis. 1975. Time, place and positional value in the chick limb-bud. J. Embryol. Exp. Morphol. 33: 621-643.

Tickle, C.A., D. Summerbell, and L. Wolpert. 1975. Positional signalling and specification of digits in chick limb morphogenesis. Nature 254: 199-202.

Turing, A.M. 1952. The chemical basis of morphogenesis. Philos. Trans. R. Soc. Lond. B. Biol. Sci. 237: 37-72.

Yost, H.J. 1990. Inhibition of proteoglycan synthesis eliminates left-right asymmetry in Xenopus larvis cardiac looping. $D e$ velopment 110: 865-874.

Zlotogora, J. and E. Elian. 1981. Asplenia and polysplenia syndromes with abnormalities of lateralization in a sibship. $J$. Med. Genet. 18: 301-302.

Zwilling, E. 1955. Ectoderm-mesoderm relationship in the development of the chick embryo limb bud. I. Exp. Zool. 128: 423-441. 


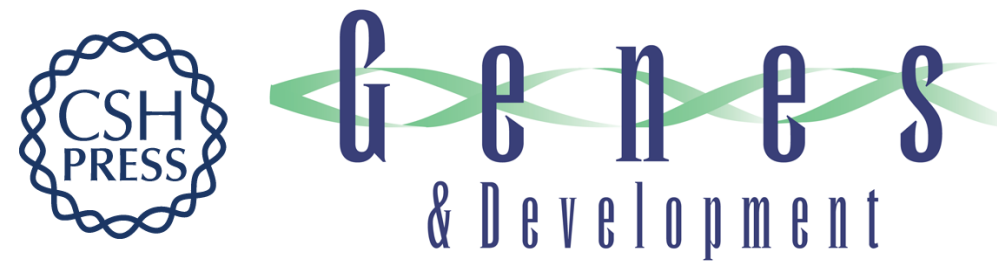

\section{legless insertional mutation: morphological, molecular, and genetic characterization.}

G Singh, D M Supp, C Schreiner, et al.

Genes Dev. 1991, 5:

Access the most recent version at doi:10.1101/gad.5.12a.2245

References This article cites 38 articles, 9 of which can be accessed free at:

http://genesdev.cshlp.org/content/5/12a/2245.full.html\#ref-list-1

License

Email Alerting

Service

Receive free email alerts when new articles cite this article - sign up in the box at the top right corner of the article or click here.

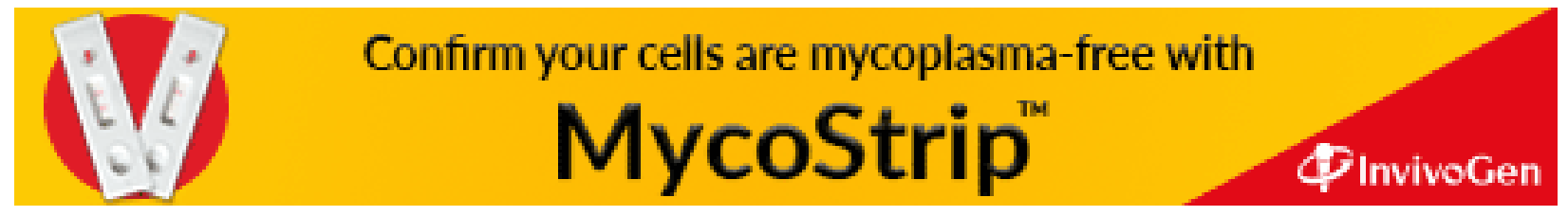

